\title{
Characteristic Analysis of Various Importance Indices for Effective Highway Network Reliability Improvement
}

\author{
Takahiro NAGAE* \\ Hiroshi WAKABAYASHI** \\ *Doctor course student, Graduate School of Faculty of Urban Science, Meijo University \\ **Professor, Faculty of Urban Science, Meijo University
}

(Received: Jul. 29, 2019 Accepted: Feb. 11, 2020)

\begin{abstract}
Threats of potential natural disasters have necessitated the urgency to construct and sustain a highly reliable network for promotion of national resilience. However, improving all the links simultaneously is difficult due to budget constraints. Therefore, network reliability can be improved effectively by improving the most important key link. Our previous research revealed that improved criticality importance $(C I W)$ is better than reliability importance $(R I)$. However, as link reliability increases, the difference between the values of both indicators shrinks in terms of fairness of link improvement. In this study, we compared four importance indices. The newly added indices are Fussell-Vesely $(F V)$ and the risk achievement worth $(R A W)$, which are used in highly reliable systems (e.g., nuclear power plants). $C I W, R I, F V$, and $R A W$ are compared by terminal reliability, difference between maximum and minimum values of link reliability (DBMM), and number of improved links. First, $R I$ and $R A W$ improve terminal reliability better compared with $C I W$ and $F V$; however, the difference is small. $R I$ and $R A W$ give a larger DBMM than $C I W$, which indicates that using $R I$ implies more gaps between parallel routes than using $C I W$ and $F V$. Moreover, $C I W$ is the best in terms of fairness of link improvement, as it has the largest number of improved links. Therefore, $C I W$ is a recommended importance index.
\end{abstract}

Keywords: Highway network reliability, Importance index, Cost-benefit analysis, Fussell-Vesely, Risk achievement worth.

\section{Introduction}

Japan is frequently struck by natural disasters, i.e., earthquake or unusual weather, potentially leading to some unusable segments of the highway and requiring a highly reliable highway network that offers alternative routes for a sure and stable traffic service. Such a scheme is called "connectivity reliability," whose improvement essentially fosters the network's anti-disaster capabilities. On the other hand, constructing highly reliable and efficient routes is subject to budget constraints and may be extremely difficult to achieve. Moreover, such an objective necessitates the establishment of an adequate quantitative index to identify the links improved in what is known as "importance analysis." Previous studies have found some issues on this matter, which are highly stressed herein as follows:

(1) When using importance indices, calculation of a network containing nine nodes and twelve links 
resembles calculation of a parallel network. Therefore, improved criticality importance $(C I W)$ is better than reliability importance $(R I)$ when analyzing the importance indices of a link in a highway network.

(2) Higher link reliability is associated with the problem of reduced difference between the indicators of indices in terms of the cost-benefit functions.

(3) In this study, in order to solve the above problem, new importance indices are added for comparison. These indices are Fussell-Vesely $(F V)$ and risk achievement worth $(R A W)$. These indices are used in high-reliability systems (e.g., aviation and nuclear power).

Moreover, necessary requirements for the importance indices of a road network are explored in this paper by newly estimating reliability improvement together with these indicators.

In this study, we simulate highway network improvement using four importance indices. As a prerequisite, it is necessary to explain existing research on highway network connectivity reliability and importance indices. Therefore, citations are made as needed within Sections $\mathbf{2}$ to $\mathbf{4}$ and Section $\mathbf{5}$ describes new research. The remainder of this paper is structured as follows: Section $\mathbf{2}$ reviews a reliability analysis of a highway network and the study of importance analysis as well as highlighting the characteristics of and problems with previously proposed importance indices. Section $\mathbf{3}$ provides a definition of connectivity reliability and explains the problems of conventional importance analyses. Section 4 presents three cost reliability functions. Section 5 compares terminal reliability improvement using four importance indices with cost-benefit functions for a network with nine nodes and twelve links. Section $\mathbf{6}$ presents concluding remarks on the problem discussed in Section 2.

\section{Review of reliability and importance indices of a highway network}

Connectivity, travel time, and capacity reliabilities (Nicholson et al., 2003) have been proposed as methods that measure the reliability of a highway network. Our study focuses on connectivity reliability and importance indices. Reliability means that "systems are in a condition to be able to accomplish a predetermined function during a prescribed period of service" (Barlow and Proschan, 1965); thus, it is defined as an expression of probability. The CPU time and the memory size required for reliability analysis increase exponentially as the size of the highway network increases. This is called a non-deterministic polynomial-time hard (NP-hard) problem. Wakabayashi and lida (1992) proposed an efficient and practical reliability analysis to solve this problem; nonetheless, efficient and practical importance analysis remains unsolved. The purpose of an importance index is to effectively improve the reliability of the system, e.g., the probability importance (Birnbaum, 1969) and criticality importance (CI) (Henley and Kumamoto, 1981) that have previously been proposed and that are discussed later. Importance indices can be interpreted as the degree to which improvement in the reliability of a component contributes to the reliability of the overall system. Moreover, in this study, the importance of the connectivity reliability of a highway network is defined as the degree to which a component contributes to the reliability between nodes when link reliability is improved. The following statements explain the problem of importance indices of a highway network as they are currently used:

(1) It is more difficult to improve a more reliable link than a less reliable link. This is termed "diminishing marginal utility." Birnbaum's importance index, $R I$, does not reflect this fact.

(2) In a parallel network, use of $R I$ and $C I$ results in only the more reliable links being improved and in less reliable links remaining unimproved.

(3) Whether proper improvement in terminal reliability is obtained by only an importance index is unknown. Therefore, considering the problem from a cost-benefit viewpoint is also necessary.

An improved criticality importance $(C I W)$ index has recently been proposed to solve problems (1) and 
(2) (Wakabayashi, 2004); nevertheless, until today, the results of applying this index to a network reveal insufficient calculation and evaluation. Furthermore, this $C I W$ index has not yet been compared with the $R I$ index, and this paper attempts to analyze this $C I W$ index and compare it with the $R I$ index. For problem (3), only a few comparisons have been made to determine what difference in terminal reliability exists when the network is improved using a conventional importance index. We can form the following hypothesis: if the cost reliability function is set such that it expresses the expense and relations of link reliability (higher link reliability), then the costs for anti-disaster measures are higher, while the expense to strengthen a link of low reliability is low. Furthermore, we developed some link reliability cost functions and compared a rational solution with an equitable solution (using a cost-benefit analysis), after which we clarified how to change using a relation with link reliability functions. Next, we established a small network and calculated the "improved path," determined by importance indices and a cost-benefit analysis. The index for comparison uses terminal reliability and the ratio of terminal reliability to cost.

On this basis, we reviewed conventional studies. A sample article regarding importance evaluation in the highway network is highlighted by a method that uses a cost-link-reliability function (Nicholson, 2007) as is described below. Nicholson (2007) proposed that link reliability be provided by the function of traffic volume; thus, the cost required for reliability improvement cannot be considered. In addition, we question whether this is beneficial because the improvement cost did not increase significantly after we compared the growth rates of the improvement cost and the link reliability.

\section{Reliability and importance indices of a highway network (Wakabayashi and Fang, 2013)}

\subsection{An overview of connectivity reliability}

This study is basically the application of reliability graph analysis (RGA) (Barlow and Proschan, 1965) to road networks. RGA analyzes the connectivity between two points of input and output by using a directed graph called a reliability block diagram for the relationship between system reliability and unit (system component) reliability. The theory underlying RGA is what is called "coherent system theory". An example of a reliability graph is shown in Figure 1.

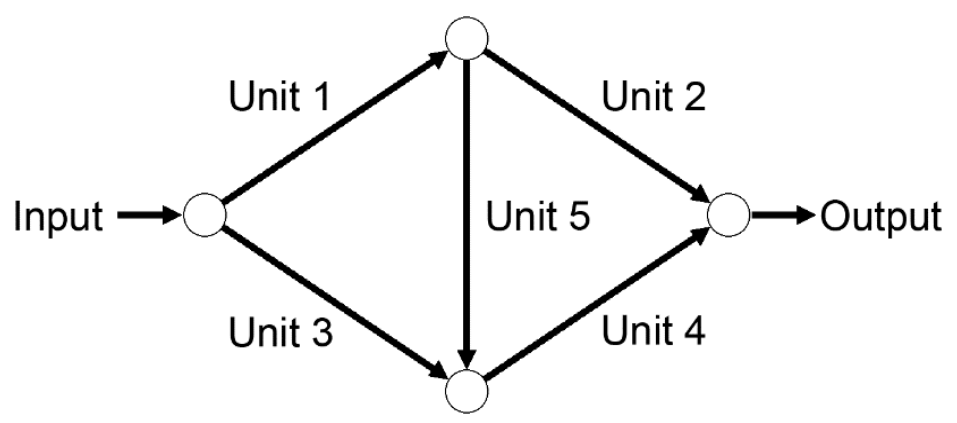

Fig. 1 An example of a reliability graph

In terms of the reliability graph, the reliability of the entire system is defined as follows:

The reliability of the entire system is the probability that all units (path set or tie set) included in at least one path between input and output will work as specified when the arrow in the figure is followed. In Fig. 1, when any one of the unit combinations $\{1,2\},\{1,5,4\}$, or $\{3,4\}$ functions simultaneously, the entire system functions. In 
this way, RGA expresses reliability using graph theory. Since the road network can also be described using graph theory, reliability graph analysis and road network reliability analysis have many common points in theoretical handling. In reliability analysis, devices composed of a relatively large number of elements (components) are collectively referred to as a system, and the components are referred to as a unit. Assume that the system and the unit have only two states: function and failure.

For unit $a$,binary variable $x_{a}$ is defined as follows:

$$
x_{a}=\left\{\begin{array}{l}
1, \text { unit } a \text { is functioning, } \\
0, \text { unit } a \text { is failing. }
\end{array}\right.
$$

With the number of units as $l$, the system state can be expressed by the following equation:

$$
\boldsymbol{x}=\left(x_{1}, x_{2}, \cdots, x_{l}\right)
$$

This vector is called a system state vector. System state $\emptyset$ can be defined using $\boldsymbol{x}$ as follows:

$$
\varnothing(x)=\left\{\begin{array}{l}
1, \text { system is functioning } \\
0, \text { system is failing. }
\end{array}\right.
$$

The structure function can know the function / failure state of the system using the state vector that represents the function / failure state of each unit. A system whose structure function has the following two properties is called a coherent system.

(i) Each unit $a(a=1, \cdots, l)$ is associated with $\emptyset(\boldsymbol{x})$.

(ii) $\emptyset(\boldsymbol{x})$ is non-decreasing for each variable $x_{a}(a=1, \cdots, l)$.

In the following, in this study, the system is a coherent system.

The system reliability is obtained as follows. The system reliability with structure function $\emptyset(\boldsymbol{x})$ is $R$, and the reliability of each unit is $r_{a}$. Define random variable $X_{a}$ for each unit as follows:

$$
X_{a}=\left\{\begin{array}{l}
1, \text { unit } a \text { is functioning, } \\
0, \text { unit } a \text { is failing. }
\end{array}\right.
$$

and

$$
\begin{gathered}
P_{r}\left\{X_{a}=1\right\}=r_{a} \\
P_{r}\left\{X_{a}=0\right\}=1-r_{a} .
\end{gathered}
$$

furthermore,

$$
E\left[X_{a}\right]=1 \times P_{r}\left\{X_{a}=1\right\}+0 \times P_{r}\left\{X_{a}=0\right\}=r_{a}
$$

A vector composed of random variables $X_{a}$ is defined by the following equation:

$$
\boldsymbol{X}=\left(X_{1}, \cdots, X_{l}\right)
$$


Function / failure of the system can be expressed by random variable $\emptyset(\boldsymbol{X})$ using structure function $\emptyset(\boldsymbol{x})$, and the system reliability is as follows:

$$
R=P_{r}\{\varnothing(\boldsymbol{X})=1\}=E[\varnothing(\boldsymbol{X})]
$$

These are definitions in reliability graph analysis. In order to divert RGA to a road network, it is necessary to make the unit correspond to the link and the system correspond to the probability of traveling between node pairs. Thus, reliability analysis of a road network can be handled in the same way as RGA.

\subsection{Connectivity reliability of a highway network}

Before we discuss our proposal of a comparative analysis for improving network reliability, we introduce the work of Wakabayashi and Fang (2013). In their work, the connectivity reliability of a highway network is defined as the probability that two given nodes within the network are connected with a certain service level of traffic for a given time period. Similarly, link reliability in the network is defined as the probability that the traffic reaches a certain service level for a given time period. However, since this study assumes an alternative route in the event of a disaster, it is simply defined as the "probability of can traveling links between the departure node to the arrival node" (travel time required is not considered).

Terminal reliability, $R$, is given by the minimal path sets expression as follows (Iida and Wakabayashi, 1988):

$$
R(\mathbf{r})=E\left[1-\prod_{s=1}^{p}\left(1-\prod_{a \in P_{s}} X_{a}\right)\right]
$$

where $P_{-} s$ is the $S$ th minimal path set, and $p$ is the number of minimal path sets. This calculation method is called the Boolean absorption method (Wakabayashi, and Iida, 1992).

Here, $\varnothing(\mathbf{X}), \mathbf{X}$ and $\mathbf{r}$ are a structural function and are vector representations for $X_{a}$ and $r_{a} . X_{a}$ is a binary indicator variable for link $a$ as:

$$
X_{a}= \begin{cases}1, & \text { if link } a \text { provides the certain service level, } \\ 0, & \text { otherwise. }\end{cases}
$$

Link reliability, $r_{a}$, is:

$$
r_{a}=E\left[X_{a}\right]
$$

In order to calculate the exact value of terminal reliability, it is necessary to use all minimal path sets. This means that the reliability value of the same link is included in the calculation process. In the following, a description will be made using a specific network. A minimal path set is a subset that involves a system failure if any one of the units (links) that make up the route from the departure node to the arrival node of the system fails. For example, in the nine-node and twelve-link network shown in Figure 2, the minimal path between node pairs $(1,9)$ are $\{1,2,5,10\},\{3,8,11,12\},\{1,4,7,10\},\{3,6,7,10\},\{1,4,9,12\},\{3,6,9,12\},\{1,2,5,7,9,12\},\{3$, $8,11,9,7,10\},\{1,4,6,8,11,12\},\{3,6,4,2,5,10\},\{1,2,5,7,6,8,11,12\}$, and $\{3,8,11,9,4,2,5,10\}$. Thus, the identical link is included in several minimal paths. To avoid overlap of probability, a Boolean absorption for the logical conjunction is required. 
The terminal reliability of a traffic network depends on the network structure and the link reliabilities. There are, therefore, two basic approaches to improving network reliability, namely, to improve the network structure or to improve the reliability of the links. The focus here is identifying which links should be improved, so as to maximize the improvement in network reliability. In this study, calculation is performed assuming that each link reliability is independent.

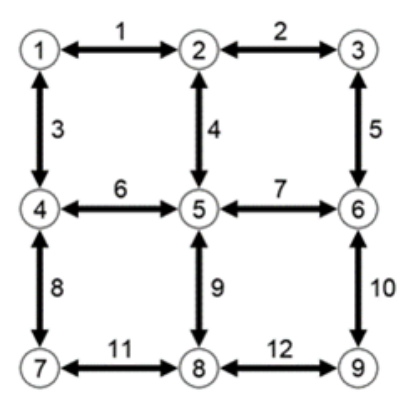

Fig.2. Nine-node and twelve-link network

\subsection{Definition of current importance indices}

To find the key links for the most efficient improvement of terminal reliability, the $R I$ index (Birnbaum, 1969) was proposed as

$$
R I_{a}=\frac{\partial R(\mathbf{r})}{\partial r_{a}}
$$

$R I$ indicates the impact of an improvement in link reliability, i.e., an increase or decrease in the reliability of link $a$ affects a corresponding increase or decrease in terminal reliability. $R I$ is also known as Birnbaum's structural importance. In Eq. (3.3.1), $R(\mathbf{r})$ is the terminal reliability between the origin node and the destination node, $a$ is the link number, and $r_{a}$ is the value of the link reliability. When it is not a specific calculation example, we abbreviate importance index $R I_{a}$ as $R I$. In the following, the same notation is used for other importance indices ( $C I, C I W, F V$, and $R A W$ ). The result from $R I$ indicates that, in the case of a parallel network, improving the more reliable link will be more effective for improving terminal reliability. Usually, however, it is difficult to improve a more reliable link, whereas it is rather easy to improve a less reliable one. This result of improving, managing, and reconstructing a network contradicts what would be expected.

The $C I$ index was proposed to counter the shortcoming of RI. By definition, it is the ratio of proportional improvement in network reliability to proportional improvement in link reliability (Henley and Kumamoto, 1992) as given by

$$
\begin{aligned}
C I_{a} & =\lim _{\Delta r_{a} \rightarrow 0}\left\{\frac{\Delta R(\mathbf{r}) / R(\mathbf{r})}{\Delta r_{a} / r_{a}}\right\} \\
& =R I_{a} \times \frac{r_{a}}{R(\mathbf{r})}
\end{aligned}
$$


The result from the $C I$ index is the same for both links in a series network, but it justifies strengthening the less reliable link. This is a shortcoming of the $C I$ index. Moreover, the index provides no information to distinguish between the two links for improving network reliability. Additionally, it indicates that, in the case of a parallel network, improving the more reliable link gives a greater increase in the terminal reliability of the network. The results for a parallel network provided by both $R I$ and $C I$ suggest that a less reliable link should be ignored.

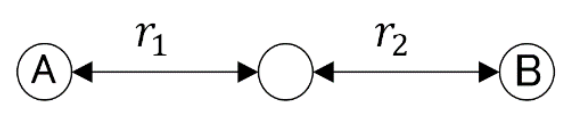

Fig. 3. Series network

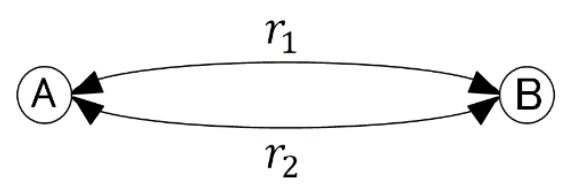

Fig. 4. Parallel network

\subsection{Definition of improved criticality importance}

Changing the definition of the equation in reliability engineering, improved criticality importance $(C I W)$, proposed by Wakabayashi (2004), is expressed by Eq. (3.4.1);

$$
\begin{aligned}
C I W_{a} & =\lim _{\Delta q_{a} \rightarrow 0}\left\{-\frac{\Delta R(\mathbf{r}) / R(\mathbf{r})}{\Delta q_{a} / q_{a}}\right\} \\
& =-\frac{\partial R(\mathbf{r})}{\partial q_{a}} \times \frac{q_{a}}{R(\mathbf{r})} \\
& =R I_{a} \times \frac{\left(1-r_{a}\right)}{R(\mathbf{r})}
\end{aligned}
$$

and

$$
q_{a}=1-r_{a}
$$

where $q_{a}\left(=1-r_{a}\right)$ is the unreliability of link $a$.Hereafter, $R$ uses the same meaning as $R(\mathbf{r})$.

$$
C I W_{a}=\frac{\left(1-r_{a}\right)}{R} R I_{a}
$$


where two links, 1 and 2, are connected in series; it follows from Eqs. (3.3.1), (3.3.2), and (3.4.1) that

$$
C I W_{1}=\frac{1-r_{1}}{r_{1}}
$$

and

$$
\operatorname{CIW}_{2}=\frac{1-r_{2}}{r_{2}}
$$

furthermore,

$$
C I W_{1}>C I W_{2} \text {, if } r_{1}<r_{2}
$$

Thus, in a series network, the $C I W$ has the same property as Birnbaum's structural importance, which is exactly that from Eq. (3.4.4), as would be expected. Similarly, where links 1 and 2 are connected in parallel, it follows from Eqs. (3.3.1), (3.3.2), and (3.4.1) that

$$
C I W_{1}=\frac{\left(1-r_{1}\right)\left(1-r_{2}\right)}{r_{1}+r_{2}-r_{1} \cdot r_{2}}=C I W_{2}
$$

Here, although $C I W$ made more progress than $C I$ proposed by Henley and Kumamoto (1981), this index is the same for both links in a parallel network, whereby it does not provide any distinguishable information between them in terms of improving network reliability. Thus, when using $C I W$, a different index is necessary for the selection of an improved link. In this paper, we consider an "improvement path" of terminal reliability using the "cost-link reliability function," which is similar to the cost-benefit ratio (Nicholson, 2007). Highly

\begin{tabular}{|c|c|c|c|}
\hline & $R I$ & $C I$ & $C I W$ \\
\hline Series network & \multicolumn{3}{|c|}{ if $r_{1}<r_{2}$} \\
\hline$(A) \stackrel{r_{1}}{\longrightarrow} \stackrel{r_{2}}{\longrightarrow}$ (B) & $R I_{1}>R I_{2}$ & $C I_{1}=C I_{2}$ & $C I W_{1}>C I W_{2}$ \\
\hline Desirable result & \multicolumn{3}{|c|}{$r_{1}>r_{2}$} \\
\hline Judgement & Good & NG & Good \\
\hline Parallel network & \multicolumn{3}{|c|}{ if $r_{1}<r_{2}$} \\
\hline$(A) \quad r_{1}=(B$ & $R I_{1}<R I_{2}$ & $\mathrm{CI}_{2}$ & $\left|C I W_{1}=C I W_{2}\right|$ \\
\hline Desirable result & \multicolumn{3}{|c|}{$r_{1}>r_{2}$} \\
\hline Judgement & NG & NG & No information \\
\hline
\end{tabular}
designated links should be chosen as important links using indices $R I, C I$, and $C I W$ for improving network reliability. See the lines of "Desirable result" in Table 1.

Table 1. Summary of the merits and demerits of three importance indices. 


\subsection{Cost reliability function and cost-benefit analysis}

As stated, a different index is required with $C I W$ for choosing an improved link. In this regard, we established the reliability of the improved link and three cost reliability functions to emphasize the relationship of the cost it requires.

(1) Case A: Type of constant - Cost

$$
\text { Cost } A=1000
$$

In this case, the cost to improve the reliability is the same regardless of the value of the link reliability.

$$
\text { Case B: Type of increase-Linear }
$$

$$
\text { Cost } B=5000 \times\left(r_{a}+0.1\right) \text {. }
$$

Here, the cost for improvement increases linearly with the link reliability.

(3) Case C: Type of increase-Quadratic

$$
\operatorname{Cost} C=500 \times\left(50 \times r_{a}^{2}+15 \times r_{a}+1\right) \text {. }
$$

In this case, the cost increases as a quadratic function of the link reliability.

The effect of an improvement in network reliability, which requires a cost increase, might not be apparent in the short term. For a long-term improvement, Eq. (3.5.4) shows a simple cost-benefit function that shows how the network reliability will improve against the cost increase:

$$
E f f(Y, F)=\frac{R-R_{0}}{\text { Cost }} \times Y \times F,
$$

where

$Y:$ Number of years to invest;

$F_{n}$ : The conversion cost-benefit of the increased traffic volume obtained by the reliability improvement in the $n$-th unit of time (the unit of time is one year);

$R_{0}$ : Original network reliability;

Cost : Cost increase to improve the network reliability from $R_{0}$ to $R$;

$E f f(Y)$ : The efficiency of the cost-benefit obtained by the reliability improvement of traffic systems in $Y$ years.

\section{Comparison of $R I$ and $C I W$ in a network consisting of nine nodes and twelve links (Nagae and Wakabayashi, 2016)}

Before the comparative analysis in Section $\mathbf{5}$, where four indices are considered, we state the characteristics of $R I$ and $C I W$ in this section. In both $R I$ and $C I W$, we calculated the dispersion of link reliability, terminal reliability, and cost-benefit analysis through a method showcasing a parallel network of nine nodes and twelve links (Fig. 2). First, we determined in the network that $R I$ had better terminal reliability improvement than $C I W$, while $C I W$ provided smaller dispersion among link reliability than RI (Wakabayashi, 2004). Second, we compared both indices. We assumed an initial value of the link reliability for six cases and the set of link reliability values by generating random variables. For this value, both cases were prepared for 100 trials, after 
which we selected the improved link yielding the maximal value of importance. We improved the value of reliability by 0.01 each time and repeated that six times. The results are shown in Table 2 and Fig.5. The results indicate that terminal reliability improvements by $R I$ are better than those determined by $C I W$. Moreover, the variance of the link reliability for $C I W$ was smaller than that for $R I$, whereas cost-benefit (Eff) results by $C I W$ were better than those determined by $R I$. From these, we could assume that $C I W$ is generally better than $R I$. However, as the link reliability value increases, the difference between $R I$ and $C I W$ decreases. Figure 5 shows how the calculation result of the total cost and Eff varies depending on the link reliability in Case C. As the initial value of link reliability increases, the cost for improvement increases, so the total cost increases for both $R I$ and $C I W$. In terms of total cost, CIW's superiority has not changed. On the other hand, with regard to Eff, the difference between $R I$ and $C I W$ narrows as the link reliability increases, and $R I$ is better than $C I W$ at $(0.8 \leqq \mathrm{r}$ $<0.9)$. In a high-reliability road network, it can be considered that the improvement in terminal reliability has a greater effect on Eff than the improvement of links with low reliability.

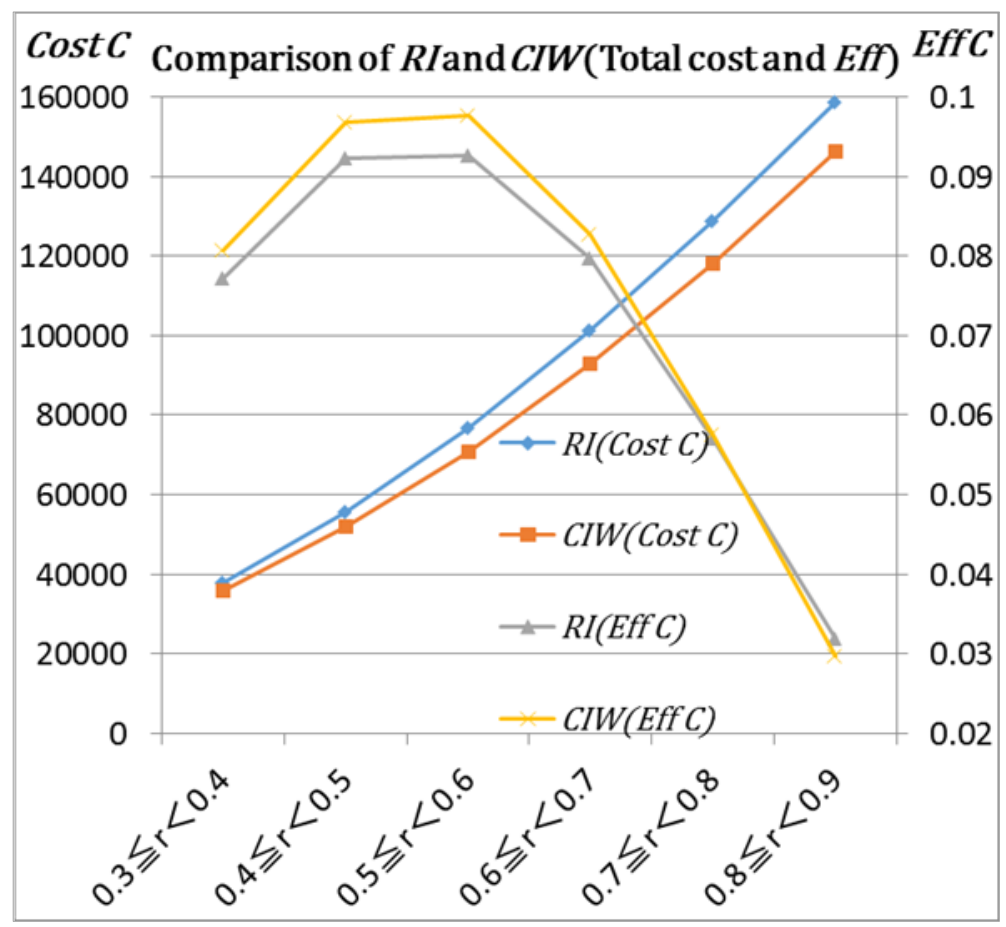

Fig. 5. Comparison of $R I$ and $C I W$ (total cost and cost-benefit function) 
Table 2. Calculation result of reliability improvement determined by $R I$ and $C I W$

\begin{tabular}{|c|c|c|c|c|}
\hline \multicolumn{2}{|l|}{$0.3 \leqq r<0.4$} & Initial value & $R I$ & CIW \\
\hline \multicolumn{2}{|c|}{ Terminal reliability $(R)$} & 0.081075 & 0.086887 & 0.086834 \\
\hline Link & $\max -\min$ & 0.084694 & 0.097449 & 0.087645 \\
\hline reliability & var & 0.000744 & 0.000903 & 0.000769 \\
\hline Link reliability & CaseB & & 12428.21 & 12027.39 \\
\hline -cost function & CaseC & & 37934.29 & 35868.34 \\
\hline Cost-benefit & CaseB & & 0.234416 & 0.239767 \\
\hline function (Eff) & CaseC & & 0.077248 & 0.080730 \\
\hline
\end{tabular}

\begin{tabular}{|ll|r|r|r|}
\hline $0.4 \leqq r<0.5$ & & \multicolumn{2}{c}{ Initial value } & \multicolumn{1}{c|}{$R I$} \\
\hline Terminal reliability $(R)$ & 0.197628 & 0.207803 & 0.207632 \\
\hline Link & $\max -\min$ & 0.084694 & 0.099669 & 0.085930 \\
reliability & var & 0.000744 & 0.000938 & 0.000741 \\
\hline Link reliability & CaseB & & 15533.63 & 14941.05 \\
-cost function & CaseC & & 55519.18 & 51870.52 \\
\hline Cost-benefit & CaseB & & 0.328121 & 0.335083 \\
function (Eff) & CaseC & & 0.092210 & 0.096762 \\
\hline
\end{tabular}

\begin{tabular}{|ll|r|r|r|}
\hline $0.5 \leqq r<0.6$ & & \multicolumn{1}{c}{ Initial value } & \multicolumn{1}{c|}{ RI } \\
\hline Terminal reliability $(R)$ & 0.374920 & 0.389087 & 0.388720 \\
\hline Link & max-min & 0.084694 & 0.103755 & 0.084733 \\
reliability & var & 0.000744 & 0.000995 & 0.000719 \\
\hline Link reliability & CaseB & & 18702.90 & 17875.81 \\
-cost function & CaseC & & 76770.99 & 70847.65 \\
\hline Cost-benefit & CaseB & & 0.379289 & 0.386219 \\
function $(E f f)$ & CaseC & & 0.092709 & 0.097611 \\
\hline
\end{tabular}

\begin{tabular}{|ll|r|r|r|}
\hline $0.6 \leqq r<0.7$ & & \multicolumn{1}{c}{ Initial value } & \multicolumn{1}{c|}{ CIW } \\
\hline Terminal reliability $(R)$ & 0.588454 & 0.604520 & 0.603825 \\
\hline Link & max-min & 0.084694 & 0.108641 & 0.084231 \\
reliability & var & 0.000744 & 0.001047 & 0.000710 \\
\hline Link reliability & CaseB & & 21860.41 & 20848.34 \\
\hline -cost function & CaseC & & 101277.18 & 93001.81 \\
\hline Cost-benefit & CaseB & & 0.367998 & 0.368869 \\
function $(E f f)$ & CaseC & & 0.079663 & 0.082801 \\
\hline
\end{tabular}

\begin{tabular}{|ll|r|r|r|}
\hline $0.7 \leqq r<0.8$ & & \multicolumn{1}{c}{ Initial value } & \multicolumn{1}{c|}{ CIW } \\
\hline Terminal reliability $(R)$ & 0.790794 & 0.805442 & 0.804356 \\
\hline Link & $\max -\min$ & 0.084694 & 0.111902 & 0.083736 \\
reliability & var & 0.000744 & 0.001090 & 0.000703 \\
\hline Link reliability & CaseB & & 24988.23 & 23829.55 \\
\hline- cost function & CaseC & & 128823.05 & 118179.58 \\
\hline Cost-benefit & CaseB & & 0.293502 & 0.284725 \\
\hline function (Eff) & CaseC & & 0.057073 & 0.057479 \\
\hline
\end{tabular}

\begin{tabular}{|ll|r|r|r|}
\hline $0.8 \leqq r<0.9$ & \multicolumn{1}{c}{ Initial value } & \multicolumn{1}{c|}{$R I$} & \multicolumn{1}{c|}{ CIW } \\
\hline Terminal reliability $(R)$ & 0.933145 & 0.943236 & 0.941806 \\
\hline Link & $\max -\min$ & 0.084694 & 0.113137 & 0.083269 \\
reliability & var & 0.000744 & 0.001102 & 0.000697 \\
\hline Link reliability & CaseB & & 28024.91 & 26810.32 \\
-cost function & CaseC & & 158689.58 & 146314.41 \\
\hline Cost-benefit & CaseB & & 0.180299 & 0.161657 \\
\cline { 1 - 2 } function $(E f f)$ & CaseC & & 0.031917 & 0.029658 \\
\hline
\end{tabular}




\section{Application of PSA importance analysis to road networks}

\subsection{Definition of the $F V$ and $R A W$ indices}

The previous sections have so far dealt with importance indices suitable for a road network, where we clearly understood the origin of the issue due to which differences between $C I W$ and $R I$ tend to decrease from the perspective of the cost-benefit functions of the link reliability. To reiterate, $C I W$ is better than RI; nevertheless, in an item for comparison, it is necessary to measure the degree of superiority or inferiority of the item as there are multiple elements describing each.

In this study, we will investigate whether other new importance indices used in high-reliability systems in other fields should be applied to road networks. In practice, importance indices are widely used in systems of aerospace, space, nuclear power, etc. These fields are characterized by complicated systems often involving a high risk of failure, where very high reliability is necessary. In particular, in the nuclear power industry, probabilistic safety assessment (PSA) is used to determine which equipment in the system is important for prevention of serious events, i.e., core damage. Herein, we estimated whether the importance index used in PSA can be used in a highly reliable road network. Table 3 summarizes the importance indices used in PSA. $Q$ (base) is the original failure probability of the whole system, $Q\left(q_{i}=1\right)$ is the failure probability of the whole system assuming that the failure probability of the target unit is 1 , and $Q\left(q_{i}=0\right)$ is the failure probability of the whole system assuming that the failure probability of the target unit is $0 . F V$ and $R A W$ are the most common importance indices in PSA. These use the notation of the citation source. " $i$ " does not include the meaning of "-th". However, " $i$ " and " $a$ " are the same in terms of one particular unit that calculates the importance. Hence, this paper treats " $i$ " as equivalent to " $a$ " of $R_{a}$. The relationship between the failure probability and the reliability can be expressed by the following equation:

$$
\begin{gathered}
q=1-r, \\
Q=1-R .
\end{gathered}
$$

The Fussell Vesely $(F V)$ index shows how low the failure probability of the entire system is; i.e., the degree to which the reliability of the system improves when the failure probability of the target unit is assumed to be 0 . In other words, a unit with a large $F V$ reflects a great effect of the improvement measures. The risk achievement worth $(R A W)$ index shows the extent to which the failure probability of the whole system increases when the failure probability of the target unit is assumed to be 1, i.e., the degree to which the reliability of the system decreases. In other words, a unit having a large value of $R A W$ indicates that contribution to reliability is high. 
Table 3. PSA importance indices

\begin{tabular}{|l|l|c|}
\hline Name & Abbr. & Formula \\
\hline Fussell-Vesely & $F V$ & $\frac{Q(\text { base })-Q\left(q_{i}=0\right)}{Q(\text { base })}$ \\
\hline $\begin{array}{l}\text { Risk reduction } \\
\text { worth }\end{array}$ & $R R W$ & $\frac{Q(\text { base })}{Q\left(q_{i}=0\right)}$ \\
\hline $\begin{array}{l}\text { Risk achievement } \\
\text { worth }\end{array}$ & $R A W$ & $\frac{Q\left(q_{i}=1\right)}{Q(\text { base })}$ \\
\hline $\begin{array}{l}\text { Birnbaum } \\
\text { importance }\end{array}$ & $\begin{array}{l}B I= \\
R I\end{array}$ & $Q\left(q_{i}=1\right)-Q\left(q_{i}=0\right)$ \\
\hline
\end{tabular}

\subsection{Significance of the $F V$ and $R A W$ indices in a road network}

$F V$ and $R A W$ have features that make them applicable to calculating the value of importance for a highly reliable road network.

First, $F V$ has similar characteristics to $C I W$ in series and parallel networks. In a series network, the degree of importance of a link with low reliability is high whereas, in a parallel network, the importance value is the same regardless of link reliability. The calculation results of $F V$ and $R A W$ in series and parallel networks is discussed below (refer to Table 3 for the calculation formula of $F V$ and $R A W$ ).

For two links in a series network (Fig. 3), terminal reliability $R$ is given by

$$
Q(\text { base })=1-\left(1-q_{1}\right)\left(1-q_{2}\right)
$$

where $Q$ (base) is system failure probability, and $q_{1}$ and $q_{2}$ are the values of failure probability for links 1 and 2 , respectively. Moreover, $Q\left(q_{1}=1\right)$ and $Q\left(q_{1}=0\right)$ become

$$
\begin{aligned}
& Q\left(q_{1}=1\right)=1-(1-1)\left(1-q_{2}\right)=Q\left(q_{2}=1\right), \\
& Q\left(q_{1}=0\right)=1-(1-0)\left(1-q_{2}\right)=q_{2} .
\end{aligned}
$$

It follows that

$$
F V_{1}>F V_{2} \text { if } q_{1}>q_{2}
$$

Therefore, in a series network, $F V$ has the same property as $C I W$. Furthermore,

$$
R A W_{1}=R A W_{2}
$$

which suggests an equal value for $R A W$ in both links, in a series network, For two links in a parallel network (Fig. 4), terminal reliability $R$ is given by 


$$
Q(\text { base })=q_{1} \cdot q_{2}
$$

Moreover, $Q\left(q_{1}=1\right)$ and $Q\left(q_{1}=0\right)$ are

$$
\begin{aligned}
& Q\left(q_{1}=1\right)=q_{2}, \\
& Q\left(q_{1}=0\right)=0=Q\left(q_{2}=0\right) .
\end{aligned}
$$

It follows that

$$
F V_{1}=F V_{2}
$$

Therefore, in a parallel network, $F V$ has the same property as $C I W$. Furthermore,

$$
R A W_{1}<R A W_{2} \text { if } q_{1}>q_{2}
$$

Next, $R A W$ is applicable as an importance index for a road network because the calculation results for series and parallel networks indicate that it has similar characteristics to $C I$; therefore, there is a problem with using $R A W$ alone. Nevertheless, as $R A W$ is defined by the expression in Table 3 , there is the characteristic that the calculation result would change greatly due to a slight difference in failure probability. In this regard, $R A W$ can be a useful index when finding important units in a highly reliable system.

In the succeeding section, we will introduce a calculation example where link reliability is improved when $F V$ and $R A W$ are utilized.

\subsection{Comparison of the $F V$ and $R A W$ indices for a network consisting of nine nodes and twelve links}

We used the $F V$ and $R A W$ indices to calculate dispersion of link reliability and terminal reliability and to conduct cost-benefit analysis, through a method for a parallel network of nine nodes and twelve links (Fig.2). Here, we assumed an initial value of the link reliability for Case $1(0.3 \leq \mathrm{r}<0.7)$, Case $2(0.4 \leq \mathrm{r}<0.6)$, Case 3 $(0.8 \leq \mathrm{r}<0.85)$ and Case $4(0.85 \leq \mathrm{r}<0.9)$ and the set of link reliability by generating random variables. For this initial value, we prepared all cases for 100 trials, after which we chose the improved link yielding a maximal value of importance. We adjusted the improvement value per time to 0.01 and repeated the improvement six times. Table 4 shows the results. 
Table 4. Calculation result of the reliability improvement determined by $R I, C I W, F V$, and $R A W$ (average of 100 trials)

\begin{tabular}{|l|l|r|r|r|r|r|}
\hline $0.3 \leqq$ & \multicolumn{2}{c}{ Initial value } & \multicolumn{1}{c}{$R I$} & \multicolumn{1}{c}{ CIW } & \multicolumn{1}{c|}{$F V$} & \multicolumn{1}{c|}{$R A W$} \\
\hline Terminal reliability $(R)$ & 0.285939 & 0.360909 & 0.353997 & 0.360909 & 0.369156 \\
\hline Link & max-min & 0.338775 & 0.428488 & 0.332619 & 0.428488 & 0.538928 \\
reliability & var & 0.011910 & 0.016829 & 0.011235 & 0.016829 & 0.022142 \\
\hline Link reliability & CaseB & & 19185.38 & 15829.26 & 15829.26 & 22373.40 \\
-cost function & CaseC & & 82421.92 & 58630.60 & 58630.61 & 107713.96 \\
\hline Cost-benefit & CaseB & & 1.985003 & 2.162511 & 2.572620 & 1.897583 \\
function(Eff) & CaseC & & 0.480095 & 0.596737 & 0.709951 & 0.409050 \\
\hline
\end{tabular}

\begin{tabular}{|c|c|c|c|c|c|c|}
\hline \multicolumn{2}{|l|}{$0.4 \leqq r<0.6$} & Initial value & $R I$ & CIW & FV & RAW \\
\hline \multicolumn{2}{|c|}{ Terminal reliability $(R)$} & 0.281817 & 0.348665 & 0.345081 & 0.357623 & 0.357771 \\
\hline Link & $\max -\min$ & 0.169387 & 0.305951 & 0.211266 & 0.211266 & 0.420757 \\
\hline reliability & var & 0.002978 & 0.007370 & 0.004249 & 0.004249 & 0.011610 \\
\hline Link reliability & CaseB & & 18815.00 & 16942.73 & 15829.26 & 21359.44 \\
\hline -cost function & CaseC & & 78362.04 & 64924.73 & 58630.61 & 98399.49 \\
\hline ost- & CaseB & & 1.782364 & 1.870238 & 2.419196 & 1.787287 \\
\hline nction (Eff) & CaseC & & 0.431773 & 0.490750 & 0.669506 & 0.391583 \\
\hline
\end{tabular}

\begin{tabular}{|l|l|r|r|r|r|r|}
\hline $0.8 \leqq$ & \multicolumn{2}{c}{ Initial value } & \multicolumn{1}{c}{$R I$} & \multicolumn{1}{c}{ CIW } & \multicolumn{1}{c|}{$F V$} & \multicolumn{1}{c|}{$R A W$} \\
\hline Terminal reliability $(R)$ & 0.904052 & 0.914819 & 0.914005 & 0.914604 & 0.916952 \\
\hline Link & max-min & 0.042347 & 0.083644 & 0.046325 & 0.067306 & 0.087509 \\
reliability & var & 0.000186 & 0.000491 & 0.000215 & 0.000345 & 0.000522 \\
\hline Link reliability & CaseB & & 27102.40 & 26275.75 & 26212.20 & 27197.77 \\
\hline -cost function & CaseC & & 149218.06 & 140995.91 & 140375.18 & 150175.34 \\
\hline Cost-benefit & CaseB & & 0.198716 & 0.189435 & 0.201323 & 0.237234 \\
function(Eff $)$ & CaseC & & 0.036115 & 0.035314 & 0.037606 & 0.042990 \\
\hline
\end{tabular}

\begin{tabular}{|l|l|r|r|r|r|r|}
\hline \multicolumn{2}{l}{ Initial value } & \multicolumn{1}{c}{$R I$} \\
\hline Terminal reliability $(R)$ & 0.955265 & 0.963202 & 0.962293 & 0.962293 & 0.964787 \\
\hline Link & max-min & 0.042347 & 0.085654 & 0.046213 & 0.044458 & 0.086443 \\
reliability & var & 0.000186 & 0.000504 & 0.000214 & 0.000199 & 0.000512 \\
\hline Link reliability & CaseB & & 28643.88 & 27773.61 & 27758.77 & 28666.04 \\
-cost function & CaseC & & 165082.00 & 155986.35 & 155833.02 & 165315.54 \\
\hline Cost-benefit & CaseB & & 0.138609 & 0.126567 & 0.126630 & 0.166163 \\
function(Eff $)$ & CaseC & & 0.024066 & 0.022543 & 0.022564 & 0.028831 \\
\hline
\end{tabular}

First, as a general trend, the numerical value of the calculation result is roughly $R I$ and $R A W$, and $C I W$ and $F V$ are similar regardless of the value of link reliability. The items compared for the calculations included, as in Section 4.2., terminal reliability, link reliability (difference between maximum and minimum, variance), values of the cost-reliability function, and cost-benefit results. With regard to terminal reliability, $R A W$ always tended towards the value of $R I$ by the best result. Similarly, no significant difference was observed for $F V$ and $C I W$. In link reliability, $C I W$ showed a good value generally, which implies that it was advantageous from the perspective of equitableness of link improvement, where this value is considered superior. However, there was a slight difference in the domain of high reliability, and $F V$ reversed $C I W$ to give the best value. Specifically, the difference between the max-min value in Case $4(0.85 \leq r<0.9)$ shows the same value up to two decimal places for both $F V$ and $C I W$. In addition, the value of var for $R I$ and $R A W$ is more than doubled; however, the value of $C I W$ and $F V$ is less than 1.2 times. Furthermore, in terms of cost, $R I$ and $R A W$ were not good overall. Cases B and $\mathrm{C}$ yielded good $C I W$ at low reliability, although at high reliability its difference from $F V$ shrank and reversed. However, in Case $\mathrm{C}(0.85 \leq \mathrm{r}<0.9)$, both $R I$ and $R A W$ cost 1.05 times or more based on $F V$, while $C I W$ is less 
than 1.004; hence, $C I W$ is not much different from $F V$. With respect to cost-benefit, $F V$ and $R A W$ gave a good value at low reliability and high reliability, respectively. At this point, the value in Case $\mathrm{C}$ with high reliability, which is considered to be the most realistic numerical value, did not reflect much difference with its numerical value in any importance indices.

Figure 6 shows the number of the links (as an improvement target) improved for 100 trials using $R I, C I W$, $F V$, and $R A W$, respectively. For example, when this number is one, the same link was improved six times. $R A W$ always selected the same link for improvement regardless of the link reliability value. Therefore, $R A W$ is inferior in view of fairness of link improvement. $R I$ often chose the same link for improvement even with low link reliability. This tendency became prominent at higher reliability, and was similar to $R A W$ in $(0.85 \leq \mathrm{r}<0.9)$. $F V$ is better in terms of fairness because the link to be improved is dispersed with low link reliability. However, the higher the link reliability, the stronger the tendency to improve the same link. This point is similar to RI. Among the four indices, $C I W$ had the largest number of improvement target links. As a striking difference against other indices, the number of improvement links was secured even at high reliability. From these results, $C I W$ is the best index in terms of fairness of link reliability improvement.
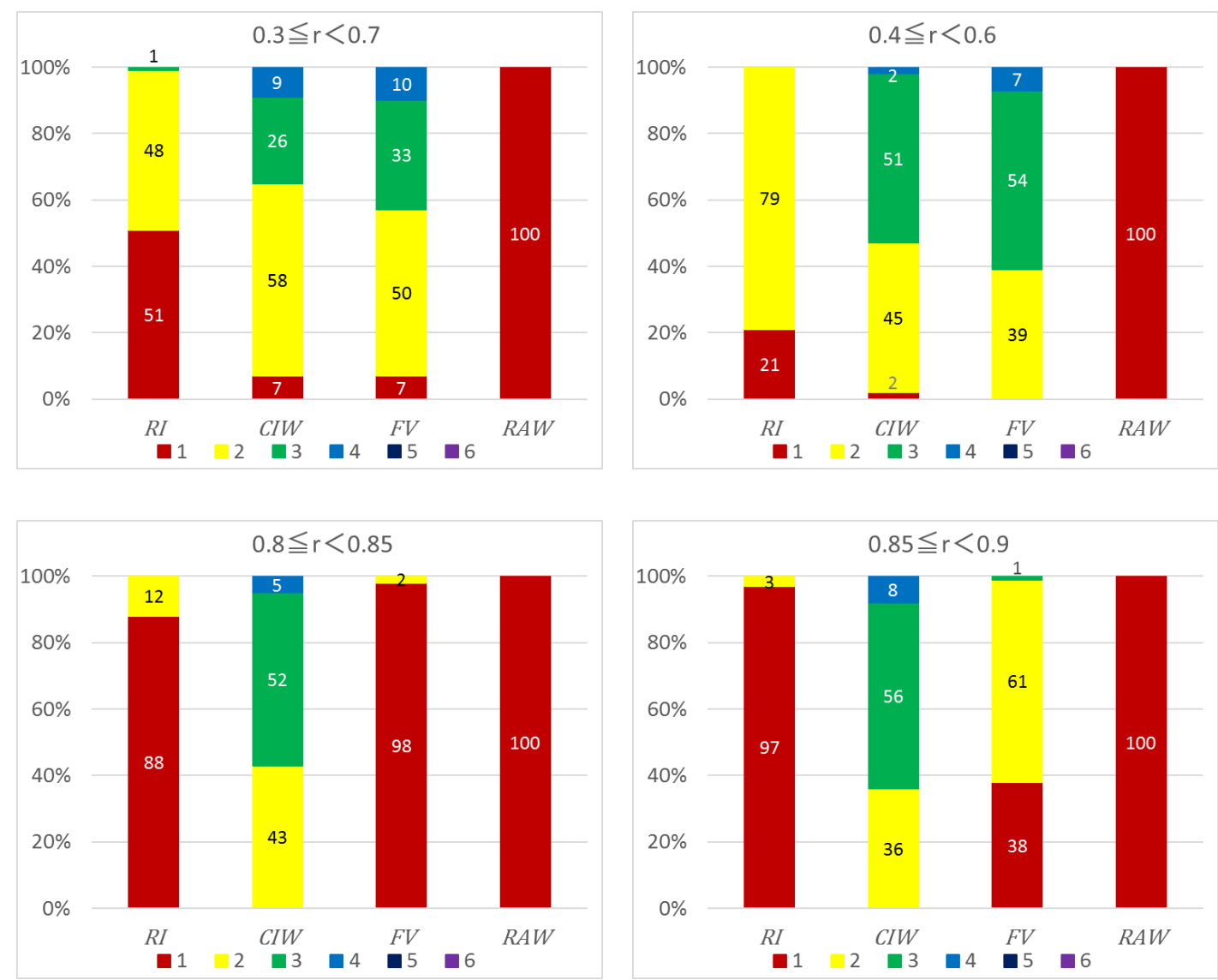

Fig. 6. Comparison of the number of improved links (RI, CIW, $F V$, and $R A W)$. 
Table 5. Merits and demerits of importance indices (RI, $C I W, F V$, and $R A W)$

\begin{tabular}{|c|c|c|c|c|c|c|c|c|c|}
\hline & & \multicolumn{2}{|c|}{$R I$} & \multicolumn{2}{|c|}{ CIW } & \multicolumn{2}{|c|}{$F V$} & \multicolumn{2}{|c|}{$R A W$} \\
\hline \multicolumn{2}{|c|}{ Range of link reliability } & Low & High & Low & High & Low & High & Low & High \\
\hline \multicolumn{2}{|c|}{ Terminal reliability $(R)$} & O & O & $\triangle$ & O & O & O & () & () \\
\hline Link & $\max -\min$ & $\triangle$ & $\triangle$ & (2) & O & O & () & $\triangle$ & $\triangle$ \\
\hline reliability & var & $\triangle$ & $\triangle$ & (2) & O & O & (2) & $\triangle$ & $\triangle$ \\
\hline \multicolumn{2}{|c|}{ Link reliability-cost } & $\mathrm{O}$ & $\triangle$ & ()) & $\mathrm{O}$ & $\mathrm{O}$ & ()) & $x$ & $\triangle$ \\
\hline \multicolumn{2}{|c|}{ Cost-benefit(Eff) } & $\triangle$ & $\mathrm{O}$ & $\mathrm{O}$ & $\triangle$ & () & $\mathrm{O}$ & $\triangle$ & () \\
\hline \multicolumn{2}{|c|}{ Number of improved links } & $\triangle$ & $x$ & 0 & (2) & $\mathrm{O}$ & $\triangle$ & $x$ & $x$ \\
\hline
\end{tabular}

\section{Conclusion}

In this paper, importance indices to improve the connectivity reliability of the highway network efficiently were examined.

First, issues related to connectivity reliability and importance indices in the highway network were organized. Next, the procedure for applying the calculation method to reliability analysis of the road network was introduced. Third, we compared three conventional importance indices with a simple network and analyzed their merits and demerits. Our previous research revealed that $C I W$ is better than $R I$ for analyzing the importance indices of a link in a highway network. However, when the reliability of the link becomes high, there arises a problem that the difference between the indicators of both is reduced in terms of cost-benefit. Therefore, in this study, other importance indices compared are $F V$ and $R A W$. Both are used in fields such as nuclear power.

As a result, $R A W$ is the best index for improving terminal reliability. For an actual high-reliability road network, $F V$ yields the best cost, while $R A W$ gives a cost-effective value. However, $F V$ and $R A W$ are inferior to $C I W$ in terms of fairness of link improvement. Overall, $C I W$ is the best for fairness of link improvement. In this regard, note that the $C I W$ index may somewhat be inferior to other indicators by virtue of total cost and cost-benefit. These results are calculation examples in a model network, and do not guarantee the same result in an actual road network. However, because it is difficult to calculate the exact value of reliability in a large-scale network, it is necessary to use an approximate calculation method. In addition, the calculation may be performed with the network shape simplified. If the network figure is similar to the nine-node and twelve-link network, the CIW index is still advantageous.

In future studies, larger and more complex networks should be simulated, and the efficiency of traffic network reliability improvement analyzed. Moreover, there is a method of giving a probability value of link reliability and conducting efficient calculation of reliability in a large-scale network. Regarding the method of estimating the link reliability value, we believe it is better to change the method according to the type of disaster. It will be easy to calculate for a certain amount of past data. One example is an assumption of flood damage due to seasonal heavy rain. For efficient calculation of network reliability, there is a method to calculate the approximate value using a partial minimal path set. The practicality of the method needs to be verified.

\section{REFERENCES}

Barlow, R.E. and Proschan, F. (1965) Mathematical Theory of Reliability. New York: John Wiley \& Sons, Inc., p. 6. 
Birnbaum, Z. W. (1969) On the importance of different components in multi-component system. In: Krishnaiah, P.R., ed. Multivariate Analysis II. New York: Academic Press, Waltham, pp. 581-592.

Henley, E.J. and Kumamoto, H. (1981) Reliability Engineering and Risk Assessment, Prentice-Hall, Inc., pp. 418-436.

Iida, Y. and Wakabayashi, H. (1988). An efficient calculation method to obtain upper and lower bounds of terminal reliability of road networks using Boolean algebra. Journal of JSCE, No. 395/IV-9, pp. 75-84 (in Japanese).

Nagae, T. and Wakabayashi, H. (2015). Differences in network reliability improvement by several importance indices. Transportation Research Procedia, Vol. 10, pp.155-165.Available online at ScienceDirect, www.sciencedirect.com. Peer-review under responsibility of Delft University of Technology, ISSN 2352-1465, doi: 10.1016/j.trpro.2015.09.065.

Nagae, T. and Wakabayashi, H. (2016). Utilization of practical importance indices for effective highway network reliability improvement, Proceeding of JSCE, No.54, CD-ROM (No.63) pp.474-481 (in Japanese).

Nicholson, A. (2007). Optimising network terminal reliability. Proceedings of the 3rd International Symposium on Transportation Network Reliability, CD-ROM.

Nicholson, A. Schmoecker, J. Bell, M.G.H. and Iida, Y (2003). Assessing transport reliability: Malevolence and user knowledge. In: Bell, M.G.H. and Iida, Y., eds. The Network Reliability of Transport, Proceedings of the 1st International Symposium on Transportation Network Reliability (INSTR), Pergamon, pp.1-22.

Wakabayashi, H. (2004). Network reliability improvement: Reliability importance. Proceedings of the 2nd International Symposium on Transport Network Reliability. University of Canterbury, Christchurch, New Zealand.

Wakabayashi, H. and Fang, S. (2013) Comparison of importance indices for highway network reliability improvement combined with cost-benefit analysis Proceedings of the 16th Meeting of the EURO Working Group on Transportation.

Wakabayashi, H. and Iida, Y. (1992). Upper and lower bounds of terminal reliability of road networks: An efficient method with Boolean algebra. Journal of Natural Disaster Science, Vol. 14, No.1, p. 29-44. 